\title{
Um Guia para Identificação e Mensuração de Dívida Técnica de Requisitos no Desenvolvimento de Software
}

\author{
Ana Carolina C. de Melo (mestranda) ${ }^{1}$ \\ Roberta Andrade de A. Fagundes (orientadora) ${ }^{1}$ \\ Wylliams B. Santos (coorientador) ${ }^{1}$ \\ ${ }^{1}$ Programa de Pós-Graduação em Engenharia de Computação \\ Universidade de Pernambuco (UPE) \\ Mestrado Acadêmico em Engenharia de Computação
}

Ingresso: agosto/2019 - Qualificação: setembro/2020 - Conclusão: agosto/2021

accm@ecomp.poli.br, \{roberta.fagundes, wbs\}@upe.br

Resumo. Contexto: Dívida Técnica (DT) descreve as consequências que os projetos de software enfrentam durante o seu desenvolvimento, ocasionada muitas vezes, quando tarefas não são realizadas adequadamente. Inicialmente, a DT possuía foco nas atividades de codificação, mas no avanço das investigações, o conceito foi ampliado nas demais fases do desenvolvimento de software, a exemplo, na engenharia de requisitos. Problema: A DT de requisitos está relacionada a distância entre o valor ideal da especificação dos requisitos e a implementação real do sistema, a qual são consequências das decisões estratégicas para ganhos imediatos, ou mudanças não propositais no contexto. Independentemente, é necessário mantê-la gerenciada para assegurar a evolução do software. Identificação e mensuração são as duas primeiras etapas do processo de gerenciamento, no entanto, na engenharia de requisitos ainda são pouco exploradas em pesquisas acadêmicas. Objetivo: Neste contexto, o principal objetivo deste trabalho é desenvolver um guia que possa auxiliar profissionais de software a identificar e mensurar a DT de requisitos existente em seus projetos. Metodologia: Para isso, a pesquisa está sendo desenvolvida através de quatro etapas. A primeira referente a coleta de evidências, objetiva identificar as informações que serão disponibilizadas no guia. A segunda, refere-se ao desenvolvimento da proposta, nela as informações identificadas anteriormente serão analisadas para na sequência o guia ser desenvolvido. Na terceira etapa referente a validação, o guia será avaliado por especialistas da área. Por fim, na quarta etapa de análise e conclusão, considerações e resultados sobre a eficácia do guia serão apresentadas. Resultados: Como resultados parciais, já foram identificadas causas que ocasionam no surgimento da DT de requisitos; estratégias existentes para auxiliar na identificação e mensuração; métricas utilizadas na etapa de mensuração; assim como dificuldades apontadas ao realizar essas atividades.

Palavras-chave: Dívida Técnica de Requisitos; Identificação; Mensuração.

Eventos Relacionados: SBES 


\section{Caracterização do Problema}

Dívida Técnica (DT) descreve as consequências que os projetos de software enfrentam durante o seu desenvolvimento, ocasionada muitas vezes, quando tarefas não são realizadas adequadamente [Rios et al. 2018]. Estas práticas estão contidas em projetos que são desenvolvidos sem conter um rigor específico quanto a sua qualidade, o que ao final, acarretam no surgimento da DT [Lim 2012]. O termo conhecido como dívida técnica foi introduzido por [Cunningham 1992]. Inicialmente, a DT possuía foco nas atividades de codificação, mas no avanço das investigações, o conceito foi ampliado para abranger as demais fases do desenvolvimento de software, a exemplo, na engenharia de requisitos [Brown et al. 2010]. Segundo Ernst (2012), uma elicitação ou análise de requisitos inadequada, ocasiona erros que elevam a incidência de DT nos projetos de software.

A DT de requisitos pode ocorrer intencionalmente, a exemplo, no caso de optar por não realizar o processo de elicitação rigorosamente; ou não intencional, nos casos em que os engenheiros de requisitos são iniciantes e podem não possuir as habilidades necessárias para realizar procedimentos técnicos e de longo prazo por exemplo [Rios et al. 2019]. Independente de como ocorra, é necessário manter a DT gerenciada para assegurar a evolução do software, evitando uma descoberta tardia de sua amplitude dispendiosa, ocasionando na incidência de juros [Alves et al. 2018].

A identificação e mensuração da dívida técnica são as duas primeiras etapas no seu processo de gerenciamento. Essas atividades são essenciais para saber qual tipo de DT existe, onde está localizada, e como estimar seu impacto no software [Alves et al. 2016]. No entanto, essas etapas na área de engenharia de requisitos ainda são pouco exploradas em pesquisas acadêmicas, e segundo Besker et al. (2018), estão entre as fases nas quais existem maiores dificuldades e complexidade na sua realização, em que a maior parte do tempo do gerenciamento é utilizado na compreensão e na medição da dívida técnica.

Neste contexto, o objetivo geral desta pesquisa é desenvolver um guia que possa auxiliar os profissionais de software a identificar e mensurar a DT de requisitos existente em seus projetos, conhecendo instruções e métricas que auxiliem a mensurar os dados necessários para a sua resolução. Para atingir o objetivo geral, objetivos específicos foram definidos, sendo eles: (i) realizar um Mapeamento Sistemático da Literatura (MSL) a fim de identificar as causas relacionadas ao surgimento da DT de requisitos; (ii) disponibilizar um conjunto de instruções e métricas que auxiliem na etapa de mensuração; (iii) conduzir estudos de caso na indústria para analisar como as empresas realizam as atividades de identificação e mensuração, a fim de relacionar as evidências da literatura com o que é utilizado na prática; (iv) desenvolver o guia de apoio e avaliá-lo com especialistas.

\section{Fundamentação Teórica}

\subsection{Dívida Técnica de Requisitos}

Para Rios et al. (2018), a DT de requisitos está relacionada à distância entre o valor ideal da especificação dos requisitos e a implementação real do sistema, a qual são consequências das decisões estratégicas para ganhos imediatos, ou mudanças não propositais no contexto, que ocasionam em custos futuros ao software. De acordo com Ernst (2012), quando os requisitos são implementados parcialmente, não efetuados, ou realizados de forma que não satisfaçam os aspectos desejáveis, ocasiona-se em exemplos reais desse tipo de DT, com destaque para problemas relacionados à segurança e desempenho. 


\subsection{Gerenciamento de Dívida Técnica}

Conforme Lim (2012), o gerenciamento da DT é um passo importante para alcançar uma boa qualidade no desenvolvimento do software, dado que, com frequência, grande parte das dívidas técnicas não são administradas. No trabalho de [Li et al. 2014], um método de gerenciamento foi proposto, contendo cinco etapas: identificação, mensuração, priorização, reembolso e monitoramento. Levando em consideração o objetivo e escopo desta pesquisa, as duas primeiras etapas serão descritas a seguir.

Identificação: abrange o processo de visualização da dívida técnica, identificando suas causas e demais atributos presentes no processo do desenvolvimento do software que levaram a sua existência. Esta atividade é crucial para uma gestão bem sucedida da DT.

Mensuração: analisa e quantifica os custos e esforços necessários para auxiliar na tomada de decisão sobre o reembolso da DT. Essas estimativas também incluem os benefícios que serão adquiridos (ou não) em corrigir a DT no momento em que foi identificada.

\section{Metodologia de Pesquisa}

Esta pesquisa está sendo desenvolvida através da condução de quatro etapas como ilustra a Figura 1, e na sequência, são apresentadas suas respectivas descrições.

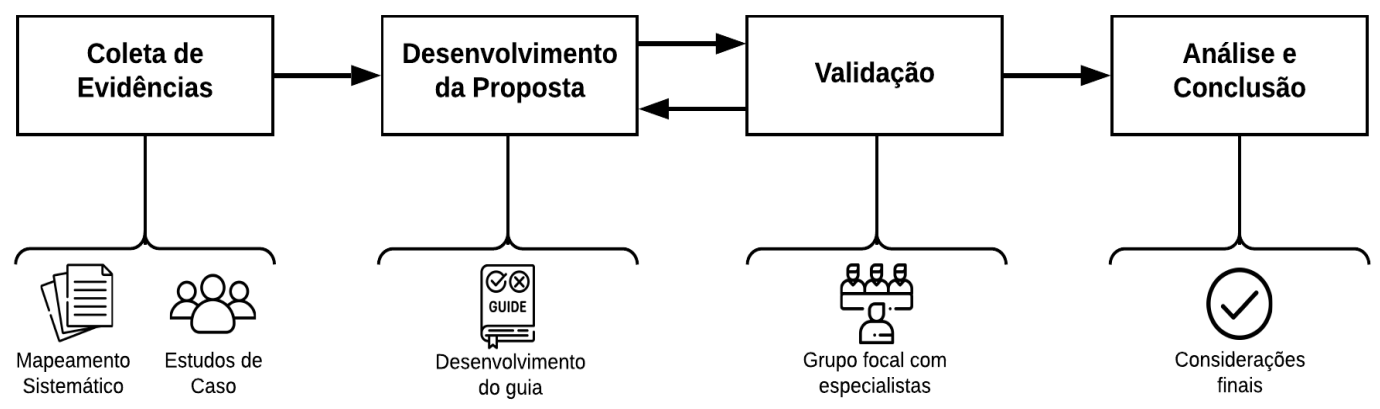

Figura 1. Etapas para Condução da Pesquisa

Coleta de Evidências: esta etapa possui como principal objetivo adquirir as informações e evidências que serão disponibilizadas no guia. Para este fim, duas atividades estão sendo realizadas, sendo elas: um MSL, e estudos de caso na indústria de software.

Desenvolvimento da Proposta: esta etapa refere-se à construção da proposta deste trabalho. Para isso, inicialmente, pretende-se relacionar as evidências obtidas no MSL com as informações relatadas e observadas nos estudos de caso. Logo após, essas informações serão parte dos inputs para o desenvolvimento do guia. Adicionalmente, também serão utilizados de base para a sua construção a ISO/IEC/IEEE 29148:2018 e a norma IEEE Std 830-1998 ${ }^{2}$. As quais são referências sobre a qualidade dos requisitos desde a sua especificação, até as demais atividades da engenharia de requisitos presentes no ciclo de vida do software. Por fim, o guia será desenvolvido, organizado em formato de technical report e, disponibilizado em um link para compartilhamento online.

\footnotetext{
${ }^{1}$ https://ieeexplore.ieee.org/document/8559686

${ }^{2} \mathrm{https} / / /$ ieeexplore.ieee.org/document/720574
} 
Validação: buscando avaliar o guia desenvolvido, pretende-se realizar um grupo focal com profissionais especialistas das áreas de engenharia de software, engenharia de requisitos e no contexto de dívida técnica, para ao final, colher as opiniões, contribuições e, sugestões de melhorias, visando validar e refinar o guia proposto para auxiliar as empresas e profissionais de software a identificar e mensurar a DT de requisitos.

Análise e Conclusão: na última etapa desta pesquisa, os resultados coletados pelo grupo focal, junto as considerações e observações colhidas no decorrer da pesquisa serão analisadas. Com isso, considerações e conclusões sobre a avaliação e eficácia do guia desenvolvido serão apresentadas, bem como explanar os possíveis trabalhos futuros.

\section{Estado Atual do Trabalho}

Dentre as etapas apresentadas anteriormente, a primeira, referente a Coleta de Evidências está em andamento e suas atividades seguem detalhadas nas subseções 4.1 e 4.2. As demais etapas não foram iniciadas, mas se encontram em processo de planejamento.

\subsection{Mapeamento Sistemático da Literatura}

O MSL encontra-se na etapa de análise e foi executado com o objetivo em conhecer os estudos disponíveis na literatura que apresentam evidências relacionadas aos anseios deste trabalho. Para a sua condução, o método proposto por [Kitchenham and Charters 2007] foi utilizado, e ao total, as etapas de planejamento, condução e relato foram consideradas. Deste modo, o MSL foi elaborado com o objetivo em responder a questão de pesquisa principal abordada nesta investigação, assim definida: "Como auxiliar na identificação e mensuração de dívida técnica de requisitos no desenvolvimento de software?”.

Para responder a essa questão, derivamos quatro subquestões especificas (QE), sendo elas: QE1 - O que tem provocado o surgimento da dívida técnica de requisitos no desenvolvimento de software? QE2 - Quais são as estratégias propostas já existentes que auxiliam na identificação e mensuração da dívida técnica de requisitos nos projetos de software? QE3 - Quais métricas estão sendo utilizadas para auxiliar no processo de mensuração? QE4 - Quais dificuldades têm sido apontadas no processo de gerenciamento da dívida técnica de requisitos no desenvolvimento de software?.

Na sequência, foram definidas as fontes e string de busca. Na busca automática, foram utilizadas as seguintes bases: IEEE Xplore; ACM Digital Library; SCOPUS; Science Direct; SpringerLink. Em seguida, uma string de busca ${ }^{3}$ foi definida para ser utilizada na condução da busca automática. Também foram realizadas buscas manuais nos periódicos e anais de conferências representativas para a área: International Workshop on Managing Technical Debt; International Requirements Engineering Conference; Journal of Systems and Software; Information and Software Technology; International Journal of Software Engineering and Knowledge Engineering.

Em seguida, foram definidos critérios de inclusão e exclusão visando préselecionar os estudos. Por fim, os critérios de qualidade do trabalho de [Dyba et al. 2007] foram utilizados como base para selecionar os estudos finais do MSL. Na segunda etapa,

\footnotetext{
${ }^{3}$ String de Busca: (( "technical debt” OR "technical debit” OR “design debt” OR “debt metaphor”) $A N D$ ("requirement” OR "requirements" OR "requirement engineering” OR "software requirements" OR "user story” "measurement” OR "measurement metrics” OR “metrics” OR “measure”))
} 
correspondente à condução da pesquisa, foi realizada a busca manual pelos estudos através do acesso aos anais das fontes de buscas. Na busca automática, os estudos foram identificados aplicando a string nas bibliotecas digitais. Ao total, 7.344 estudos foram retornados das buscas, 204 foram pré-selecionados, e ao final, 56 estudos foram considerados, os quais seguem disponíveis online ${ }^{4}$. Por fim, na última etapa, estão sendo utilizados o software ATLAS.ti e uma planilha eletrônica para analisar as evidências extraídas para responder as questões de pesquisa, que seguem apresentadas na Seção 5.

\subsection{Estudos de Caso}

Com o objetivo em analisar na prática como as empresas de software realizam o processo de identificação e mensuração da DT de requisitos, estudos de caso serão realizados em contextos reais. As informações serão colhidas por meio de entrevistas semi-estruturadas com profissionais, além de observações feitas em campo. Como objeto de estudo, empresas de desenvolvimento de software do porto digital pernambucano serão utilizadas, as quais estão sendo selecionadas considerando a existência da DT em seus projetos. Para isso, está sendo levado como critério adotado o perfil das equipes e profissionais. Tornase necessário que os mesmos já tenham conhecimento sobre DT e, que exista experiência prática sobre o seu gerenciamento, em especial relacionada a identificação e mensuração.

Como auxilio na condução dos estudos de caso, o trabalho proposto por Runeson et al. (2012) será utilizado como guideline, o qual propõe etapas para a sua realização, e que servirão de base para este trabalho, sendo elas: (i) desenho do estudo de caso; (ii) preparação para a coleta de dados; (iii) coleta dos dados; (iv) análise dos dados; (v) relatórios. Ao final, os estudos de caso serão realizados para responder às seguintes questões de pesquisa: QP1 - Quais as causas que os profissionais de software atribuem ao surgimento da DT de requisitos? QP2 - Quais estratégias que os profissionais de software já utilizam para identificar e mensurar a DT de requisitos? QP3 - Como os profissionais de software realizam os cálculos na etapa de mensuração? QP4 - Quais as principais dificuldades que os profissionais de software enfrentam ao gerenciar a DT de requisitos?

\section{Resultados Preliminares}

Durante os meses de março a junho de 2020 as primeiras etapas do MSL foram realizadas. Assim, alguns resultados preliminares já foram identificados por questão de pesquisa.

QE1: Foram identificadas 35 causas que podem levar ao surgimento da DT de requisitos nos projetos de software. Dentre as mais citadas estão: (i) Falta de comunicação com as partes interessadas; (ii) Pressão de cronograma, reduzindo o tempo para realização das atividades; (iii) Elicitação mal conduzida, ocasionando em requisitos vagos e incompletos; (iv) Falta de experiência dos profissionais; e (v) Entrevistas mal planejadas e realizadas.

QE2: Ao total, 18 estratégias para auxiliar a identificar e mensurar a DT de requisitos foram identificadas, destaque a: (i) Gerenciamento automatizado, utilizando ferramentas e softwares; (ii) Template para documentação da DT; (iii) Revisão com o cliente, auxiliando a identificar possíveis DTs, pois o feedback fornece conhecimento sobre a necessidade de correção nos requisitos especificados; (iv) Mesclar análise manual e automatizada.

\footnotetext{
${ }^{4}$ http://bit.ly/ListaEstudosFinais
} 
QE3: Foram identificadas métricas que auxiliam na mensuração das atividades de: (i) Principal, juros e probabilidade de juros (relacionados ao reembolso da DT); (ii) Decidir qual o melhor período de tempo para reembolsar a dívida técnica de requisitos.

QE4: Ao total, 22 dificuldades relatadas nos estudos referentes ao processo de gestão da DT foram identificadas, destaque para: (i) Equilibrar os benefícios de resolver a DT com os custos associados a esse processo; (ii) Destinar mais tempo e esforço para ser gasto na elicitação dos requisitos; (iii) Falta de colaboração do cliente nesse processo; (v) Conseguir que toda a equipe esteja incluída no processo de gerenciamento da DT.

\section{Contribuições}

Apesar de parciais, os resultados mostram uma quantidade considerável de informações relevantes ao objetivo desta dissertação, com isso, como principais contribuiçõos esperase: (i) disponibilização de um guia de fácil compartilhamento para auxiliar profissionais de software a identificar e mensurar DTs de requisitos em seus projetos; (ii) ampliar a compreensão de tópicos relacionados a dívida técnica e seu processo de gerenciamento, permitindo que profissionais possam identificar como conteúdos específicos desses assuntos podem ser fragmentados; (iii) fornecer relatos de experiência em contextos reais da indústria de software por meio da condução dos estudos de caso, permitindo relacionar com as informações relatadas na literatura acadêmica, para ao final, ofertar informações sólidas e avaliadas por especialistas na área. (iv) a partir da identificação das dificuldades que são encontradas ao realizar o gerenciamento da DT, se tornar uma oportunidade para o desenvolvimento de novas pesquisas e tecnologias para essa área.

\section{Comparação com Trabalhos Relacionados}

O trabalho de [Lenarduzzi and Fucci 2019] tinha o objetivo de fornecer uma definição holística da dívida técnica de requisitos. Para isso, foram utilizadas evidências da literatura, junto a um estudo exploratório composto por entrevistas e grupo focal para entender o que os profissionais de software consideram sobre esse tipo de DT, e ao final, compararam essas perspectivas com os conceitos disponíveis na literatura.

Na pesquisa de [Alves et al. 2018], foi desenvolvida uma abordagem de gestão para DT de requisitos, para isso, utilizaram da revisão sistemática para detectar os métodos já existentes de identificação, medição e gestão. Logo após, através da pesquisaação, aplicaram a abordagem em uma empresa de telecomunicações. Ao final, os resultados mostraram que os atributos usados aumentaram a visibilidade desse tipo de DT.

No trabalho de [SOUSA 2016], foi elaborado um mapa de apoio para auxiliar na gestão de DT presente nas atividades de teste de software. Para isso, evidências da literatura foram mapeadas a fim de obter as informações que seriam disponibilizadas, e na sequência, avaliadas por meio de um survey por profissionais. Logo após, foi elaborado o mapa de apoio, o qual ao final, foi avaliado por três profissionais da área de teste.

Todos os trabalhos citados e a presente proposta de mestrado, se relacionam por buscarem formas de compreender e gerenciar tipos específicos de DT. No caso de [SOUSA 2016], a proposta se assemelha por ofertar um manual de apoio, formado por evidências da literatura e, validado por especialistas, um dos diferenciais se torna o tipo de DT abordado. Nos trabalhos de [Lenarduzzi and Fucci 2019] e [Alves et al. 2018], 
ambos focaram na DT de requisitos, mas não abordaram conteúdos específicos sobre as causas para o seu surgimento, métricas para a mensuração, além de ofertar o conteúdo de forma acessível a profissionais que desejam utilizar dessas informações em seus projetos.

\section{Referências}

Alves, M., Nunes, Gava, V., and Luiz (2018). Uma proposta para identificar, medir e gerenciar a dívida técnica em requisitos de software. International Conference on Information Systems and Technology Management.

Alves, N. S., Mendes, T. S., de Mendonça, M. G., Spínola, R. O., Shull, F., and Seaman, C. (2016). Identification and management of technical debt: A systematic mapping study. Information and Software Technology, 70:100-121.

Besker, T., Martini, A., and Bosch, J. (2018). Technical debt cripples software developer productivity.

Brown, N., Cai, Y., Guo, Y., Kazman, R., Kim, M., Kruchten, P., Lim, E., MacCormack, A., Nord, R., Ozkaya, I., et al. (2010). Managing technical debt in software-reliant systems. In Proceedings of workshop on Future of software engineering research.

Cunningham, W. (1992). The wycash portfolio management system. ACM SIGPLAN OOPS Messenger, 4(2):29-30.

Dyba, T., Dingsoyr, T., and Hanssen, G. K. (2007). Applying systematic reviews to diverse study types: An experience report. In First International Symposium on Empirical Software Engineering and Measurement (ESEM 2007), pages 225-234. IEEE.

Ernst, N. A. (2012). On the role of requirements in understanding and managing technical debt. In 2012 Third International Workshop on Managing Technical Debt (MTD).

Kitchenham, B. and Charters, S. (2007). Guidelines for performing systematic literature reviews in software engineering.

Lenarduzzi, V. and Fucci, D. (2019). Towards a holistic definition of requirements debt. In 2019 ACM/IEEE International Symposium on Empirical Software Engineering and Measurement (ESEM), pages 1-5. IEEE.

Li, Z., Liang, P., and Avgeriou, P. (2014). Architectural debt management in valueoriented architecting. In Economics-Driven Software Architecture, pages 183-204.

Lim, E. (2012). Technical debt: what software practitioners have to say. PhD thesis, University of British Columbia.

Rios, N., de Mendonça Neto, M. G., and Spínola, R. O. (2018). A tertiary study on technical debt: Types, management strategies, research trends, and base information for practitioners. Information and Software Technology, 102:117-145.

Rios, N., Spínola, R. O., Mendonça, M., and Seaman, C. (2019). Supporting analysis of technical debt causes and effects with cross-company probabilistic cause-effect diagrams. In 2019 IEEE/ACM International Conference on Technical Debt (TechDebt).

Runeson, P., Host, M., Rainer, A., and Regnell, B. (2012). Case study research in software engineering: Guidelines and examples. John Wiley \& Sons.

SOUSA, C. L. d. (2016). Mapa de apoio à gestão de dívida técnica no processo de teste de software. Master's thesis, Universidade Federal de Pernambuco. 